\title{
Correction to: Tracing Rhizophagus irregularis isolate IR27 in Ziziphus mauritiana roots under field conditions
}

\author{
Babacar Thioye $^{1,2} \cdot$ Dirk Redecker $^{3} \cdot$ Diederik van Tuinen $^{3} \cdot$ Aboubacry Kane $^{1} \cdot$ Sergio Mania de Faria ${ }^{4}$. \\ Dioumacor Fall ${ }^{5}$. Diaminatou Sanogo ${ }^{5}$ - Cheikh Ndiaye ${ }^{1} \cdot$ Robin Duponnois $^{2} \cdot$ Samba Ndao Sylla ${ }^{1}$. \\ Amadou Mustapha Bâ ${ }^{2,6}$
}

Published online: 6 February 2020

(C) Springer-Verlag GmbH Germany, part of Springer Nature 2020

Correction to: Mycorrhiza (2019) 29:77-83

https://doi.org/10.1007/s00572-018-0875-3

The authors of "Tracing Rhizophagus irregularis isolate IR27 in Ziziphus mauritiana roots under field conditions" (Thioye, B., van Tuinen, D., Kane, A. et al. Mycorrhiza (2019) 29: 77. https://doi.org/10.1007/s00572-018-0875-3) inadvertently omitted Dirk Redecker, Agroécologie, AgroSup Dijon, CNRS, INRA, Université Bourgogne Franche-Comté, F-21000, Dijon, France, Dioumacor Fall and Diaminatou Sanogo, Centre National de Recherches Forestières, Dakar, Senegal, from the list of authors.

The online version of the original article can be found at https://doi.org/ 10.1007/s00572-018-0875-3

Babacar Thioye

babacarthioye@yahoo.fr

1 Laboratoire Commun de Microbiologie IRD/ISRA/UCAD, BP 1386 Dakar, Senegal

2 Laboratoire des Symbioses Tropicales et Méditerranéennes UMR113 INRA/AGRO-M/CIRAD/IRD/UM2-TA10/J, Campus International de Baillarguet, 34398 Montpellier, France

3 Agroécologie, AgroSup Dijon, CNRS, INRA, Université Bourgogne Franche-Comté, F-21000, Dijon, France

4 Embrapa Agrobiologia, Km 7 BR 465, Seropedica, Rio de Janeiro 23890 000, Brazil

5 Centre National de Recherches Forestières, Dakar, Senegal

6 Laboratoire de Biologie et Physiologie Végétales, Faculté des Sciences Exactes et Naturelles, Université des Antilles, BP 592, 97159 Pointe-à-Pitre, Guadeloupe, France
The revised author group is now: Babacar Thioye, Dirk Redecker, Diederik van Tuinen, Aboubacry Kane, Sergio Mania de Faria, Dioumacor Fall, Diaminatou Sanogo, Cheikh Ndiaye, Robin Duponnois, Samba Ndao Sylla and Amadou Mustapha Bâ.

Please use this authorship list when citing this article.

Publisher's Note Springer Nature remains neutral with regard to jurisdictional claims in published maps and institutional affiliations. 